\title{
IMPLEMENTAÇÃO DA ESTRATÉGIA DE ENSINO-APRENDIZAGEM À FAMÍLIA DE PACIENTE CRÔNICO
}

\author{
THE IMPLEMENTATION OF TEACHING-LEARNING STRATEGY FOR FAMILIES OF CHRONIC \\ PATIENTS
}

\author{
IMPLEMENTACIÓN DE UNA ESTRATEGIA DE ENSEÑANZA-APRENDIZAJE A LAFAMILIA DEL \\ PACIENTE CRÓNICO
}

Maria Célia de Freitas ${ }^{1}$

Mary Elizabeth de Santana ${ }^{2}$

RESUMO: O estudo objetivou implementar a estratégia de ensino-aprendizagem para familia de paciente em condição crônica, momento considerado ideal para reavaliar suas necessidades e as habilidades para administrar tal condição, assim como prepará-la para o cuidado no domicilio. Realizou-se uma avaliação diagnóstica de familiar/acompanhante, durante o cuidado na hospitalização de uma pessoa com insuficiência cardíaca congestiva, identificando-se o diagnóstico de enfermagem: "déficit de conhecimento". Assim, elaborou-se um plano de ensino para essa cuidadora, com o fim de prepará-la para o cuidado domiciliar. Durante a implementação, realizaram-se avaliações formativas visando conhecer a eficácia das estratégias empregadas e a somativa no domicilio. Constatou-se, portanto, o quanto é importante para o enfermeiro recorrer à estratégia de ensino-aprendizagem para orientar o familiar/cuidador no processo do cuidar de pessoas em condição crônica, com a finalidade de promover a vida, apesar da doença, uma vez que a qualidade desse cuidado prevenirá recidivas agudizantes e conseqüentes reinternações.

PALAVRAS-CHAVE: ensino-aprendizagem, diagnóstico de enfermagem, cuidador familiar, condição crônica

ABSTRACT: This study aims at implementing the teaching-learning strategy for families of patients in chronic conditions. It considers the importance of evaluating the needs and abilities of family members in order to prepare them to manage that situation appropriately, as well as to be able to provide adequate home care. A diagnostic evaluation of a family member/ caretaker was conducted during the hospitalization of a patient with congestive cardiac insufficiency. During the diagnosis "knowledge deficit" was identified. Thus, a teaching plan was elaborated for such caretaker with the purpose of preparing $\mathrm{him} / \mathrm{her}$ for home care. During implementation, formative evaluations were conducted with the purpose of learning about the efficacy of the strategies employed and the summative in the patient's home. The importance of nurses' resorting to the teaching-learning strategy in order to guide the family member/caretaker in the process of caring for people with chronic conditions was certified. The aim is promoting life, in spite of the disease, since the quality of such care will prevent aggravating recurrence and consequent re-hospitalizations.

KEYWORDS: teaching-learning, nursing diagnosis, family caretaker, chronic condition

RESUMEN: El estudio tuvo por objeto implementar una estrategia de enseñanza-aprendizaje a la familia de un paciente en condición crónica, momento considerado ideal para evaluar sus necesidades y la habilidad para administrar dicha condición, asi como prepararla para el cuidado en su propio domicilio. Se realizó una evaluación diagnóstica del familiar/acompañante, durante el cuidado de la hospitalización de una persona con insuficiencia cardiaca congestiva, identificándose el diagnóstico como: "déficit de conocimiento". Así, se elaboró un plan de enseñanza para esa cuidadora, a fin de prepararla para el cuidado domiciliar. Durante la implementación, se realizaron evaluaciones formativas para saber la eficacia de las estrategias empleadas y la sumativa en el domicilio. Se constató por lo tanto, cuan importante es para el enfermero recurrir a la estrategia de enseñanza-aprendizaje para orientar al familiar/cuidador en el proceso de cuidar a las personas en condición crónica, con la finalidad de promover la vida, a pesar de la enfermedad, una vez que la calidad de ese cuidado va a prevenir recidivas agudizantes y consecuentes reingresos en el hospital.

PALABRAS CLAVE: enseñanza-aprendizaje, diagnóstico de enfermeria, cuidador familiar, condición crónica

Recebido em 03/10/2001

Aprovado em 26/06/2002

\footnotetext{
${ }^{1}$ Doutoranda em Enfermagem Fundamental da Escola de Enfermagem de Ribeirão Preto-USP. Professora da Universidade Estadual do Ceará. Enfermeira do Instituto Dr. José Frota.

${ }^{2}$ Doutoranda em Enfermagem Fundamental de Escola de Enfermagem de Ribeirão Preto-USP. Professora da Universidade Federal do Pará e Universidade do Estado do Pará.
} 


\section{INTRODUÇÃO}

A educação do paciente é uma experiência planejada e prevista para estimular mudanças em seu comportamento e em seu estado de saúde. Na educação do paciente, o enfermeiro, dentre os profissionais de saúde, pode usar uma combinação de métodos e estratégias que possibilitem a modificação do comportamento do mesmo, em relação às condutas, para promover a saúde.

Como o ambiente está em constante mudança o ensino não deve apenas informar, mas "facilitar a mudança e a aprendizagem" (LIBÂNEO, 1994, 127).

Neste caso, ensino pode ser entendido como meio sistematizado de introduzir informações novas, eventos, experiências ou objetos no ambiente do paciente; é uma forma de comunicação distinta, estruturada unicamente e seqüenciada para produzir aprendizagem. Teoricamente, o ensino deve atender às necessidades do paciente quanto à aquisição de informações para adotar experiências novas.

O processo de ensino é compreendido de acordo com o desenvolvimento e transformação da capacidade intelectual do aprendiz, sendo direcionado aos objetivos definidos para os domínios cognitivo, afetivo e psicomotor, implicando em passos gradativos conforme os critérios preestabelecidos, tais como: idade, preparo do paciente, habilidades, interesse e motivação. Assim, a aprendizagem poderá ser demonstrada pela mudança de comportamento do individuo. E de acordo com suas necessidades educativas, é que os enfermeiros fornecerão informações, instruções e recursos à familia e amigos, disponiveis ao aprendizado.

A educação é um processo que beneficia o paciente, auxiliando-o ao alivio de ansiedade e medos, no conhecimento de sua doença e debilidade, favorecendo sua compreensão para o enfrentamento positivo da mesma. Também o beneficia na aceleração do retorno ao convivio familiar, na retomada das atividades desenvolvidas anteriormente, na redução do custo com a hospitalização; na elevação da sua auto-estima pelo aumento da efetividade do tratamento e desaparecimento das sintomatologias, na prevenção de queixas acerca dos cuidados, redução da dor imediata ou residual percebida, além de desenvolver suporte aos esforços daqueles que the prestam cuidados.

O cuidado domiciliar baseia-se na filosofia do cuidado, que é um processo interativo entre o cuidador (membro da família ou amigo) e o ser cuidado (paciente). Nele, o cuidador tem a função ativa para desenvolver ações ou comportamentos de cuidar, enquanto o ser cuidado é passivo em função de sua condição, podendo contribuir com o cuidador desempenhando um papel menos passivo, e tornando-se responsável pelo próprio cuidado em situações de educação para a saúde (WALDOW, 1998).

Outro aspecto importante do cuidado é a participação e o envolvimento da familia no cuidar do paciente portador de condição crônica, como neste caso de insuficiência cardiaca congestiva descompensada. Esta doença crônica é definida como a incapacidade cardiaca em bombear sangue suficiente para atender às necessidades teciduais de oxigênio e nutrientes, acometendo as câmaras cardiacas direita e esquerda, levando a pessoa a episódios de debilidades e necessidades de ajuda profissional e familiar (SMELTZER; BARE, 2000).
Familia é entendida, aqui, como a célula primária e o refúgio onde o indivíduo ameaçado se protege durante $o$ periodo em que terá de efetuar mudanças em seu estilo de vida, em decorrência do recente diagnóstico de doença crônica, precisando para isso de tempo para absorver o significado da doença e o impacto dela na sua vida. Ao interagirem, paciente e familiar-cuidador, formam um todo, amplo e diferente da soma dos membros individuais. Por esse motivo é importante que o enfermeiro esteja consciente de suas próprias definições e percepções acerca da estrutura e funcionamento familiar, ao efetuar a coleta de dados junto à familia e paciente, evitando, assim, hiatos e distorções das observações e interações realizadas.

Deve-se considerar que o contato do enfermeiro com o familiar-cuidador, no caso a esposa, ainda no hospital e, posteriormente, no domicilio são ocasiões ideais para reavaliar as necessidades, a habilidade de administrar o problema de saúde e também para fornecer informações adicionais sobre o controle da condição crônica à manutenção e reabilitação da vida saudável, apesar da doença (BORDENAVE; PEREIRA, 1998).

O ensino em saúde, portanto, é uma atividade que permite ao enfermeiro adotar na prática do seu quotidiano a seleção, organização e explicação dos conteúdos, de modo a direcionar as atividades de ensino, descrevendo objetivos, métodos, formas organizativas e meios adequados em favor do bem-estar do paciente, aliando-se ao familiar-cuidador.

O estudo que ora se apresenta enfatiza relato de experiência junto à familiar-cuidadora (esposa) e tem como objetivo: implementar a estratégia de ensino-aprendizagem à família de paciente institucionalizado, em condição crônica.

\section{METODOLOGIA}

Realizou-se o presente estudo na unidade de clínica de um hospital do Municipio de Fortaleza-CE, junto a um paciente e sua família (esposa), que se encontravam institucionalizados.

No seu desenvolvimento, consideraram-se as Diretrizes e Normas Regulamentadoras da Pesquisa Envolvendo Seres Humanos, Resolução 196/96 (BRASIL,1996) para obtenção do consentimento livre e esclarecido, dos participantes, os quais foram informados pelos pesquisadores sobre o objetivo da pesquisa, a importância de contribuirem com a mesma, ausência de risco, para eles, sendo-lhes assegurado o anonimato.

A coleta de dados aconteceu nos meses de setembro e outubro/2000, periodo da institucionalização; após a alta a coleta continuou com a visita domiciliar. A escolha do participante familiar recaiu sobre a esposa por ser ela quem ficava mais tempo junto ao paciente, fazia os questionamentos e relatava as anormalidades ocorridas no domicilio.

Inicialmente, fez-se uma avaliação informal (interação e observação) com a finalidade de estabelecer laços de confiança e conhecer as necessidades mais evidentes que permitissem organizar ações imediatas e a longo prazo. Este tipo de avaliação é o melhor caminho para reunir dados preliminares referentes às necessidades de aprendizagem, tanto do paciente quanto da familia, uma vez que a educação do paciente e o planejamento para a alta são funções vitais 
Implementação da estratégia...

para a ênfase nos cuidados domiciliares e na redução da permanência no hospital.

Após essa etapa, retomou-se a coleta de dados formais com entrevista, tendo como roteiro as categorias: necessidades psicossocial, espiritual e psicobiológica da familia (esposa) e paciente, com objetivo de se identificar o diagnóstico de enfermagem (DE), planejar e elaborar as intervenções que atendessem ao paciente e ajudassem o familiar-cuidador no pós-alta.

Com a finalidade de facilitar o entendimento ao leitor, apresenta-se, a seguir, o caso clínico da pessoa cuidada. Senhor de 70 anos, casado, 11 filhos, aposentado, mulato, $1^{\circ} \mathrm{grau}$ incompleto, nível sócio-econômico baixo e portador de miocardiopatia chagásica.

Admitido na unidade de emergência com história de dispnéia, aos mínimos esforços, onde permaneceu internado durante oito dias. Apresentava tosse produtiva, hipotensão arterial, anasarca e cianose de extremidades. Após avaliação médica foi encaminhado ao Unidade de Terapia Intensiva (UTI) com o diagnóstico de insuficiência cardiaca congestiva descompensada.

Exame físico: Apresentava dispnéia aos mínimos esforços, tosse produtiva, hipotensão arterial, anasarca e cianose de extremidades. $T=36.8^{\circ} \mathrm{C} ; \mathrm{FC}=73 \mathrm{bpm} ; \mathrm{R}=28 \mathrm{mpm}$ e $\mathrm{PA}=90 \times 60 \mathrm{mmHg}$.

No nono dia de internação, teve alta da UTI, sendo encaminhado à clínica médica, onde continuou recebendo oxigênio por meio de cateter nasal (1//min), hidratação venosa em veia periférica em membro superior esquerdo e sonda vesical de demorada. Apresentava dispnéia, tosse com expectoração amarelada e espessa, posição de tripé, oligúria e membros inferiores com discreto edema. $T=36.5^{\circ} \mathrm{C}$; $\mathrm{FC}=72 \mathrm{bpm} ; \mathrm{R}=22 \mathrm{mpm}$ e $\mathrm{PA}=110 \times 60 \mathrm{mmHg}$.

Para identificar os diagnósticos de enfermagem (D.E) os pesquisadores utilizaram o raciocínio de Risner (1986), para quem o diagnóstico é um processo de tomada de decisão que considera a coleta de dados (interação, observação e mensuração) para a interpretação dos mesmos numa determinada situação, fundamentada no conhecimento científico e experiência prática (Quadro 1).

Quadro 1- Análise dos dados da entrevista

\begin{tabular}{|c|c|c|c|}
\hline $\begin{array}{l}\text { AGRUPAMENTO } \\
\text { "...estou preocupada, com meu } \\
\text { marido, não sei o que vai } \\
\text { acontecer, desta vez (...) ele } \\
\text { está internando muitas vezes... } \\
\text { Estou esperançosa que chegue } \\
\text { uma resposta, prá ver ser eu } \\
\text { vou agüentar (...) de novo. Novo } \\
\text { internamento. Que doença é } \\
\text { essa? } \\
\text { "... gostaria de ter informações } \\
\text { sobre os exames, doença (...) } \\
\text { não sei nada, nem ele, só } \\
\text { obedece. Será que vão operar? } \\
\text { Será que ele vai agüentar?, } \\
\text { Está tão cansado (falta de ar), } \\
\text { olha unhas roxas!!!. }\end{array}$ & $\begin{array}{l}\text { COMPARAÇÃO } \\
\text { Ausência de } \\
\text { preocupação em } \\
\text { relação à } \\
\text { doença, } \\
\text { tratamento e } \\
\text { cuidados. Ter } \\
\text { conhecimento } \\
\text { sobre a doença, } \\
\text { tratamento e } \\
\text { cuidados }\end{array}$ & $\begin{array}{l}\text { INFERÊNCIA } \\
\text { Desconhecime } \\
\text { nto sobre o } \\
\text { problema de } \\
\text { saúde, do } \\
\text { marido. } \\
\text { Desconhecime } \\
\text { nto da própria } \\
\text { alteração de } \\
\text { saúde }\end{array}$ & $\begin{array}{l}\text { RELAÇÃO } \\
\text { Verbalização } \\
\text { de não } \\
\text { conhecer o } \\
\text { que é a } \\
\text { doença e o } \\
\text { motivo de } \\
\text { repetidas } \\
\text { internações } \\
\text { em curtos } \\
\text { períodos de } \\
\text { tempo e a } \\
\text { justificativa } \\
\text { para a "falta } \\
\text { de ar" do } \\
\text { paciente. }\end{array}$ \\
\hline
\end{tabular}

Elaborou-se o diagnóstico de enfermagem de acordo com a taxonomia da North American Nursing Association (NANDA,2000).

O D.E. identificado foi déficit de conhecimentos (doença e condição crônica), relacionado a não-familiaridade com os recursos de informação e limitação cognitiva, manifestado pela verbalização do problema, tanto por parte do familiar-cuidador como do paciente(NANDA ,2000).

O desconhecimento às vezes, é a causa da ansiedade em paciente e familiares, porisso, os sinais de déficit de conhecimento são observados através de comportamentos inadequados, imprecisão no seguimento das informação e exacerbação freqüente da doença.

Priorizou-se o referido D.E, porque acreditou-se que a alteração da saúde com a presença de doença crônica altera também a trajetória de vida não somente do paciente, mas de toda a família e que a aquisição de conhecimentos com fins de reabilitar e manter a saúde favorece a elaboração de estratégias para o enfrentamento positivo e adaptação à nova condição de vida.

Ao concluir a fase de identificação do D.E. passouse ao planejamento, com estabelecimento de metas, objetivos, estratégias, implementação e avaliação.

Em seguida, elaborou-se um plano de ensino com objetivo de preparar a esposa para o cuidado domiciliar.

\section{PLANEJAMENTO}

META

Entender o que é a doença, suas alterações e como adotar medidas preventivas de complicação. 


\section{OBJETIVOS}

- Falar sobre a doença, insuficiência cardiaca congestiva descompensada.

- Citar três sinais de alterações/complicações.

- Aprender como manusear materiais e equipamentos necessários à oxigenação.

- Descrever vantagens de seguir as prescrições médicas e de enfermagem.

\section{ESTRATÉGIAS}

- Observar a disponibilidade da esposa e paciente para se implementar a estratégia de ensino.

- Permitir que o paciente e esposa expressem suas dúvidas e conhecimentos sobre a doença.

- Estabelecer um relacionamento de comunicação efetiva que desperte a confiança.

- Descreve a doença, órgão atingido, complicações, atividades e esforços físicos permitidos, como também restrições.

- Ensinar o uso correto do cateter e do cilindro de oxigênio, conforme necessidade do paciente, no dia-a-dia.

- Estimular a repetição dos conhecimentos apreendidos.

- Avaliar os resultados objetivos do aprendizado, após a estratégia de ensino.

IMPLEMENTAÇÃO

Em uma sala, organizaram-se papéis, cartazes, lápis, revistas e cola. Foi solicitado ao paciente e esposa que descrevessem o coração. Poderiam desenhar, colar figura, escrever ou colar palavras. A partir dessa aproximação associou-se o conhecimento científico ao senso comum, permitindo a compreensão. Nesse momento, os pesquisadores descreveram sobre o coração, sua função, a doença (ICC), necessidade de limitações de alguma atividades, restrições alimentares e a possivel ocorrência de dispnéia (falta de ar), sua justificativa e a relação com o coração e a doença. Em seguida, solicitaram que reconhecessem e citassem pelo menos uma das complicações, para que reconhecessem se alcançaram a meta e objetivos.

Essa atividade teve a duração de 19 dias, sendo realizada na própria instituição, com duração de 30 minutos, incluindo avaliações formativas semanais e somativas. Posteriormente, foram feitas duas visitas ao domicílio, após a alta.

No domicilio, constataram-se adaptações na estrutura do quarto do paciente, assim como a utilização das orientações dadas durante a internação.

A esposa demonstrou como manuseava cateter e cilindro de oxigênio e como administrava os medicamentos orais e inalatórios, dizendo seguir os horários estabelecidos na prescrição médica. Informou que o esposo queixava-se menos dos sintomas da doença e colaborava na ingestão de medicamentos e com a dieta. Contudo, estava preocupada com o uso contínuo de oxigênio e com a presença de dispnéia ao menor esforço

Quadro 2 - Plano de ensino

\begin{tabular}{|c|c|c|c|c|c|}
\hline DATA & $\begin{array}{l}\text { OBJETIVOS } \\
\text { ESPECIIFICOS }\end{array}$ & CONTEÚDO & ESTRATÉGIAS & RECURSOS & $\begin{array}{l}\text { REFERÊNCIA } \\
\text { BIBLIOGRÁFICA }\end{array}$ \\
\hline \multirow[t]{2}{*}{ 28/09/00 } & $\begin{array}{l}\text { Falar sobre a } \\
\text { doença (ICC) }\end{array}$ & $\begin{array}{l}\text { Importância e } \\
\text { função do } \\
\text { coração }\end{array}$ & \multirow{4}{*}{$\begin{array}{l}\text { Papéis, } \\
\text { cartazes, lápis, } \\
\text { cola e } \\
\text { revistas }\end{array}$} & \multirow[t]{4}{*}{$\begin{array}{l}\text { Quadro } \\
\text { negro e } \\
\text { giz }\end{array}$} & \multirow[t]{4}{*}{$\begin{array}{l}\text { SMELTZER, S.C; } \\
\text { BARE, B.C.(2000). }\end{array}$} \\
\hline & $\begin{array}{l}\text { Enumerar } 3 \\
\text { sinais de } \\
\text { complicações }\end{array}$ & $\begin{array}{l}\text { Citar } \\
\text { complicações } \\
\text { da doença } \\
\text { (ICC) }\end{array}$ & & & \\
\hline \multirow[t]{2}{*}{ 29/09/00 } & $\begin{array}{l}\text { Aprender como } \\
\text { manusear } \\
\text { materiais e } \\
\text { equipamentos } \\
\text { necessários } \\
\text { para } \\
\text { oxigenação }\end{array}$ & $\begin{array}{l}\text { Materiais e } \\
\text { Medicamento } \\
\text { s utilizados } \\
\text { na } \\
\text { oxigenioterap } \\
\text { ia e como } \\
\text { manusear } \\
\text { esses } \\
\text { materiais e } \\
\text { equipamento } \\
\text { s }\end{array}$ & & & \\
\hline & $\begin{array}{l}\text { Descrever as } \\
\text { vantagens de } \\
\text { Seguir as } \\
\text { prescrições }\end{array}$ & $\begin{array}{l}\text { Tratamento } \\
\text { na ICC. }\end{array}$ & & & \\
\hline
\end{tabular}




\section{CONSIDERAÇÕES FINAIS}

A hospitalização constitui-se em um momento singular, por proporcionar a avaliação e reavaliação das necessidades e habilidades da família e do paciente, quanto ao cuidado aos obstáculos da vida e da doença.

É importante que os profissionais de saúde, dentre eles o enfermeiro, recorram à estratégia de ensinoaprendizagem, para transmitir informações a pacientes e familiares, no processo de cuidar de pessoas no domicílio, principalmente quando estas são acometidas por doenças que desencadeiam cronicidade, uma vez que tal condição altera a trajetória de vida não somente da pessoa, mas também de seus familiares.

Quando os profissionais se dispõem a utilizar essa estratégia que tem por finalidade promover a vida apesar da doença/condição crônica, consideram que a qualidade desse cuidado prevenirá recidivas, exacerbações de complicações com conseqüentes reinternações.

Considera-se, pois, fundamental que essas atividades educativas, direcionadas aos pacientes e familiares, façam parte das atividades práticas dos alunos de graduação, quando em contato com pacientes, nas diferentes instituições de saúde.

O ensino voltado ao cuidado da família e paciente para referente à aprendizagem no domicilio deve ser vislumbrado, ainda na fase de formação dos alunos de enfermagem (graduação), para facilitar sua habilidade na elaboração de planos de ensino e a escolha de estratégias, implementação e avaliação do cuidado, posto que na aplicação do processo de enfermagem a identificação dos diagnósticos de enfermagem poderá viabilizar a necessidade desse recurso.

\section{REFERÊNCIAS BIBLIOGRÁFICAS}

BORDENAVE, J.D; Pereira, A.M. Estratégias de ensinoaprendizagem. 16. ed. Petrópolis(RJ): Vozes, 1998.

LIBÂNEO, M.T. Didática. São Paulo: Cortez, 1994.

BRASIL. Ministério da Saúde. Diretrizes e normas regulamentadoras de pesquisa envolvendo seres humanos. Resolução n 196, de 10 de outubro de 1996. Brasília: Ministério da Saúde, 1996.

NORTH AMERICAN NURSING ASSOCIATION - NANDA. Diagnóstico de enfermagem: definições e classificação, 1999/ 2000. Porto Alegre: ArtMED, 2000.

RISNER, P.B. Diagnosis analysis and syntheseis of data. In: CHRISTENSES P. J. et al. Nursing process: aplication of conceptual model. 3.ed. St. Louis : Mosby, 1986.

SMELTZER, S.C; BARE B.G. Tratado de enfermagem médicocirúrgica. 8.ed. Rio de Janeiro: Guanabara Koogan, 2000.

WALDOW, V.R. Cuidado humano: o resgate necessário. Porto Alegre: Sagra Luzzatto, 1998. 